Review Paper

\title{
Exploitation of Battery Energy Storage in Load Frequency Control -A Literature Survey
}

\author{
Ahmed Zurfi and Jing Zhang \\ Department of Systems Engineering, University of Arkansas at Little Rock, Little Rock, AR, USA
}

\author{
Article history \\ Received: 04-10-2016 \\ Revised: $18-10-2016$ \\ Accepted: 13-12-2016 \\ Corresponding Author: \\ Ahmed Zurfi \\ Department of Systems \\ Engineering, University of \\ Arkansas at Little Rock, Little \\ Rock, AR, USA \\ Email: ajabbas@ualr.edu
}

\begin{abstract}
Deregulation of the electricity market and the continued attempts by the international community to reduce the effects of global climate change have resulted in a rapid increase in the usage of different renewable forms of energy such as the wind and solar energy. The availability of such renewables is highly sporadic in nature. Consequently, their integration into the power system is a practice not without unfavorable impacts on the stability of the power system. The stability of the power system frequency is vulnerable to sudden imbalances between generation and demand. Therefore, the intermittent generation from the renewables imposes undesirable frequency fluctuations. Load Frequency Control (LFC) is the control scheme responsible for restoring system frequency to its rated value after any generation-demand imbalance that causes the frequency to deviate from the rated value. Therefore, efficient and fast LFC techniques are crucial. Utilization of Battery Energy Storage (BES) is one of the techniques applied to improve the performance of LFC due to its distinctive characteristics of fast response and high energy density. This paper is aimed to provide a succinct and comprehensive literature survey of the different concepts and strategies of the utilization of battery energy storage systems for LFC services. The study was conducted by reviewing, collating and interpreting numerous research papers on the relevant topics. Based on the BES scale, location, control, ownership and coordination with the system operator, the existing strategies are classified into two main categories: Grid-Scale Systems (GSS) and Load-Scale Systems (LSS). The LSS category is subclassified as Coordinated Aggregate Systems and Non-Coordinated Individual Systems. The survey theoretically discussed the advantages and disadvantages of each category from a technical perspective.
\end{abstract}

Keywords: Load Frequency Control, Battery Energy Storage, Vehicle-toGrid, Demand Side Control, Smart Loads, Primary Frequency Control

\section{Introduction}

The balance between generation and demand must always be preserved during the steady-state operation of interconnected power systems. Maintaining the generation-demand balance is responsible for keeping the system frequency and tie-line power interchange between control areas at their scheduled values. Otherwise, severe frequency and tie-line power deviations, if not timely suppressed, will substantially impact the stability of the power system. The imbalance between the generation and demand within the power system can take place at any instant by variations on either the generation side, when one or more generation unit is tripped or on the demand side when large loads are added to the system. Such variations in the load could randomly happen during the day and they are hard to expect even with a precise short-term load forecasting (Zhao, Topcu, and Low 2013) (Saxena and Hote 2013; "Balancing and Frequency Control" 2011). 
The percentage of the stochastic power generation by the renewables is reaching considerable levels in today's power system (Beiter 2014). As a result, the system is undergoing challenging frequency fluctuations. Generally, "balancing authorities", as they are defined by the North American Electric Reliability Corporation (NERC), maintain generation-demand balance to regulate the frequency by applying three control schemes that are the primary, secondary and tertiary control and each scheme reacts within a specified period of time. Figure 1 shows a diagram of the time windows of each control scheme ("Balancing and Frequency Control" 2011; Galus, Koch, and Anderson 2011). The secondary control or Load Frequency Control (LFC) is an essential control strategy and its major purpose is to adjust the power generation of a certain control area to maintain the system frequency and tie-line power interchange at predefined values during steady-state operation (Bakken and Grande 1998; Kundur 1993). Typically, LFC embodies the "minute-to-minute" control that restores the line frequency to its scheduled value following any disturbance during the day ("Balancing and Frequency Control" 2011).

The conventional LFC strategy is achieved via control of different types of online generation units that are synchronized with the system and participate partially in supplying the demand (Jaleeli et al. 1992). A control signal defined as the Area Control Error (ACE) is derived from the sum of the frequency deviation and the deviation of tie-line power interchange. The ACE signal is sent by the Automatic Generation Controller (AGC) to the LFC units to adjust their generation in order to keep this error minimized (Kundur 1993). Consequently, this trend of LFC faces inevitable economic and practical challenges: Increased operational and maintenance costs of the system, and reduced efficiency of generation units due to partial-load operation (Leitermann and Kirtley 2010; Samarakoon, Ekanayake, and Jenkins 2012; Short, Infield, and Freris 2007). Furthermore, LFC responds in a time window of 1-10 minutes due to machines inertial response which, under certain operational conditions, is not sufficiently fast ("Balancing and Frequency Control" 2011). Therefore, many scholars have discussed various control strategies to improve the performance of load frequency control to withstand fast and sudden mismatches between the generation and demand.

One practice for enhancement of the performance of LFC is to utilize fast-responsive energy storage sources which can absorb such rapid disturbances introduced to the system. Various energy storage technologies including high power flywheels, pumped storage hydro, compressed air, superconducting magnetic storage, and battery storage can be enlisted to support power systems stability in different techniques (Joseph and Shahidehpour 2006). Battery energy storage systems (BESS) are considered one of the significant options owing to their competitive features such as high energy density and fast dynamic response (Lu, Liu, and Wu 1995; Amano et al. 2012; Ribeiro et al. 2001). These features along with developments in power electronics converters paved the way for BESS to play a noticeable role in frequency regulation applications (Chowdhury and Zaman 2014).

An abundance of work has been reported on the utilization of BESS in LFC. The authors in (Pandey, Mohanty, and Kishor 2013) have presented an exhaustive review on LFC and highlighted the concerns of various power system models on the dynamics of LFC. They also discussed LFC of systems integrate renewable energy sources, FACTS as well as BESS. However, a lack of details about the utilization of BESS in LFC can be noticed. This paper attempts to review the different categories of the existing strategies of LFC with BESS, compares their merits and pitfalls and discusses their technical aspects. The rest of the paper is structured as follows. Section 2 presents a general background about the role of battery energy storage technologies in power system applications. The classification of the existing strategies of LFC-BESS is presented in section 3. The conclusions are presented in section 4 .

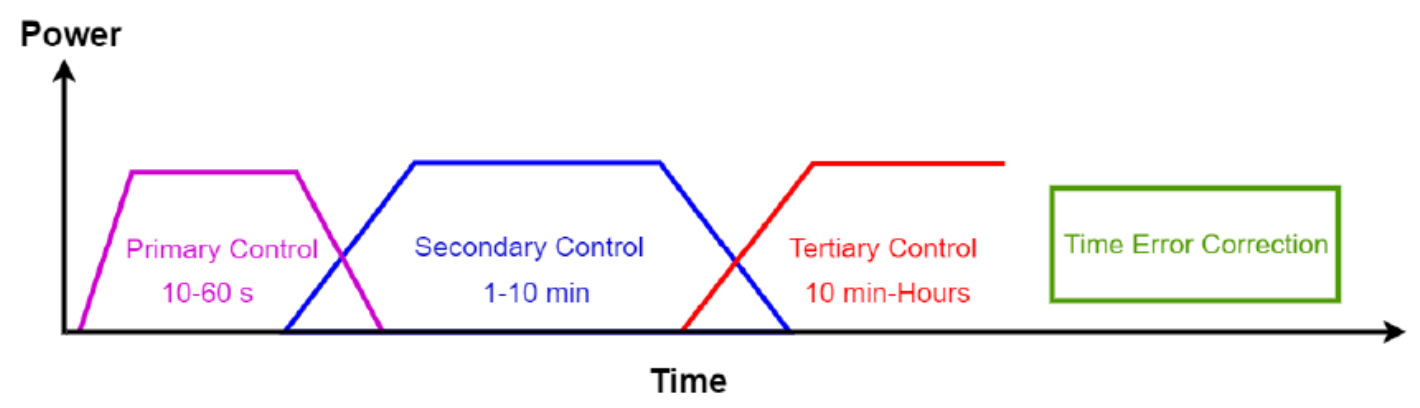

Fig. 1. Time windows of the frequency control schemes (BFC, 2011; Galus et al., 2011) 


\section{Background}

Battery energy storage technologies in power system applications have been in operation since the early 1900s. The authors in (Goldsbrouch and Fansler 1903) discussed the use of batteries as an equalizer and implemented the tests on the distribution substations that supplied the Union Traction Company of Indiana in 1902. BES systems can be exploited in a wide range of grid support applications including generation, transmission, and distribution as well as end-user services owing to their fast response to track sudden power fluctuations (Akhil 1995; "Grid Energy Storage" 2013; Cha et al. 2012). For instance, it was reported in (Lian et al. 2014) that - for frequency regulation - it could be 2-3 times more effective to use energy storage than adding a combustion generation unit to the system. BESS are found in numerous applications such as peak shaving (Rahimi et al. 2013; Alam, Muttaqi, and Sutanto 2013; Alexandre Oudalov, Cherkaoui, and Beguin 2007), load leveling (Papi 2006 ; Jung, Kim, and Rho 1996; Mehr, Masoum, and Jabalameli 2013), black start support (W. Liu et al. 2016; Dong et al. 2014; Noce et al. 2012), frequency regulation (Serban and Marinescu 2014; Li et al. 2014; Khalid and Savkin
2010), load shedding (S. Zhang et al. 2013; Fadrhonc, Matamoros, and Sood 2014), and voltage support (Agrawal et al. 2014; Bhatt and Chowdhury 2011). A hierarchical summary of utilization of battery energy storage in power systems is shown in Figure 2. Load frequency control is one of these applications, and it has been employing BESS for decades now. Provision of BESS in LFC is being discussed by researchers since the mid-1980s after Kunisch et al. proposed the first effective BES demonstration facility as an approach to improve the performance of LFC control in Germany-1986 (Kunisch, Kramer, and Dominik 1986; Lu, Liu, and Wu 1995; Kottick, Blau, and Edelstein 1993). BESS can improve the LFC and regulate system frequency by consuming energy (charging) during periods when the frequency is above the scheduled value and providing energy (discharging) back to the electric grid when the frequency drops below scheduled value under control of LFC scheme. Till present, it is considered an inspiring topic for researchers particularly as the achievement of smart grids concept has recently been expanded (Ghiassi-farrokhfal, Keshav, and Rosenberg 2015; Martínez and Serna 2015).

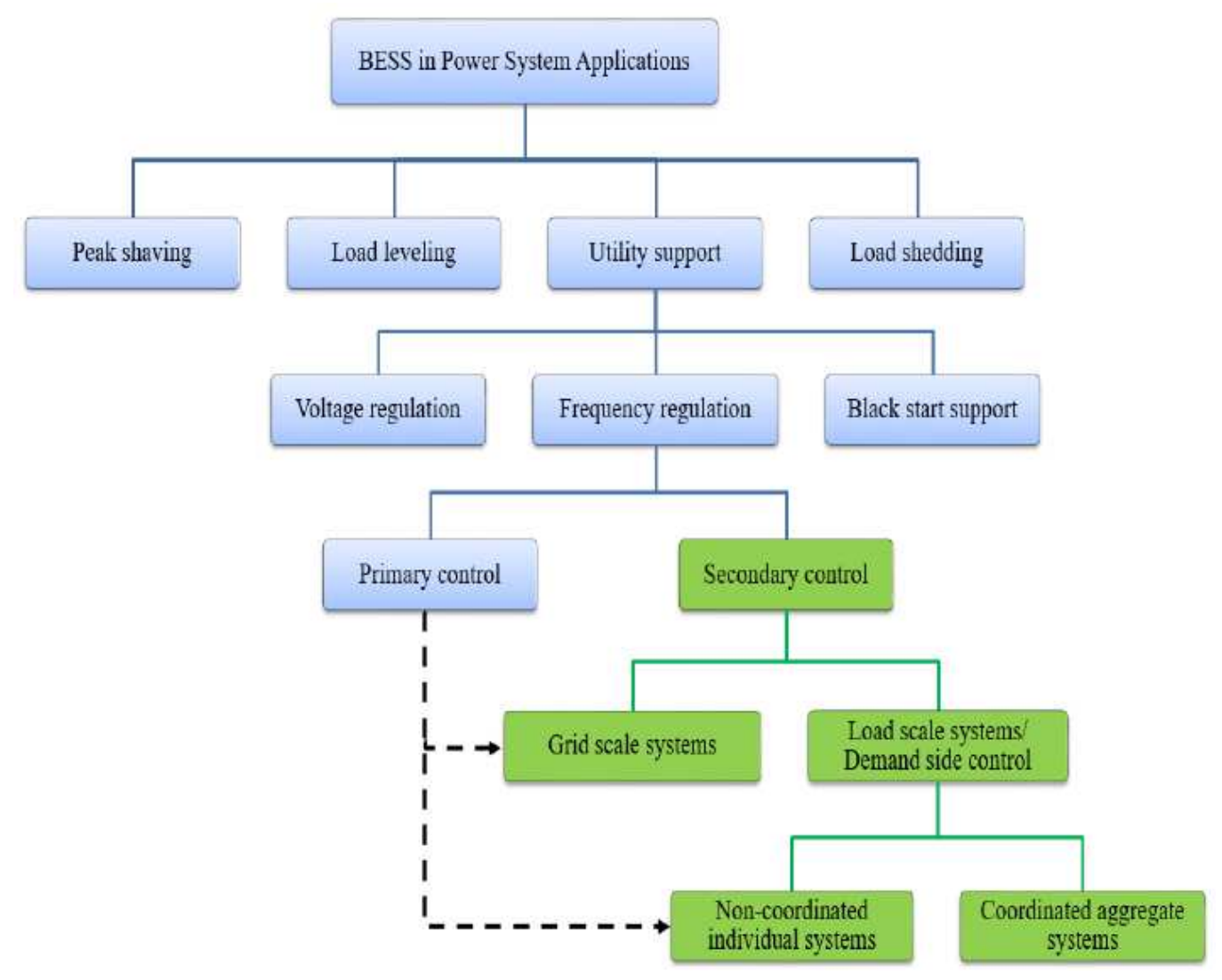

Fig. 2. A hierarchical diagram of the different applications of BESS in power systems 


\section{Utilization of BESS in LFC}

The concept of the LFC control strategy with the utilization of BES can be explained in the block diagram shown in Fig. 3 (Taisuke Masuta, Yokoyama, and Tada 2010). In this work, the strategy is mainly classified into two categories based on the scale of the battery energy storage, its ownership and location in the power system, its asset holding and the way it coordinates with Independent System Operators (ISO) or dispatching center (these two terms will be used interchangeably in the next discussions). The two categories are defined as Grid-Scale Systems (GSS) and Load-Scale Systems (LSS).

\section{Grid-Scale Systems}

This is the conventional trend where centralized battery storage facilities are installed in the power system and controlled by LFC. Authors in (Aditya and Das 2001)) have discussed the application of BES for LFC of a two area interconnected power system with reheat thermal plant considering the generation rate constraint for modern reheat type. According to their simulation results, a significant improvement in system response has been obviously noticed under $1 \%$ step increase in the demand. Likewise, (Kalyani, Nagalakshmi, and R 2012) and (Sen and Kumar 2014) have discussed the effectiveness of BES for LFC of a two area system through Matlab simulation. In isolated power grids where no interconnection to a large power system is available, the necessity for reliable frequency regulation is more significant because of the small inertia spinning reserve (Kottick, Blau, and Edelstein 1993). In (Aditya and Das 1999), the improvement of the LFC performance of an isolated system was achieved using BESS. The effect of the BESS on LFC of an isolated power system has also been studied in (Mercier, Cherkaoui, and Oudalov 2009) with considering the optimal sizing of the BESS. The effect of BESS has been investigated for a case of study of one month of operation through a simulated system. Results showed that frequency deviation of $-0.98 \%$ (scheduled $50 \mathrm{~Hz}$ ) was recorded without BESS while it was $-0.26 \%$ with using BESS.

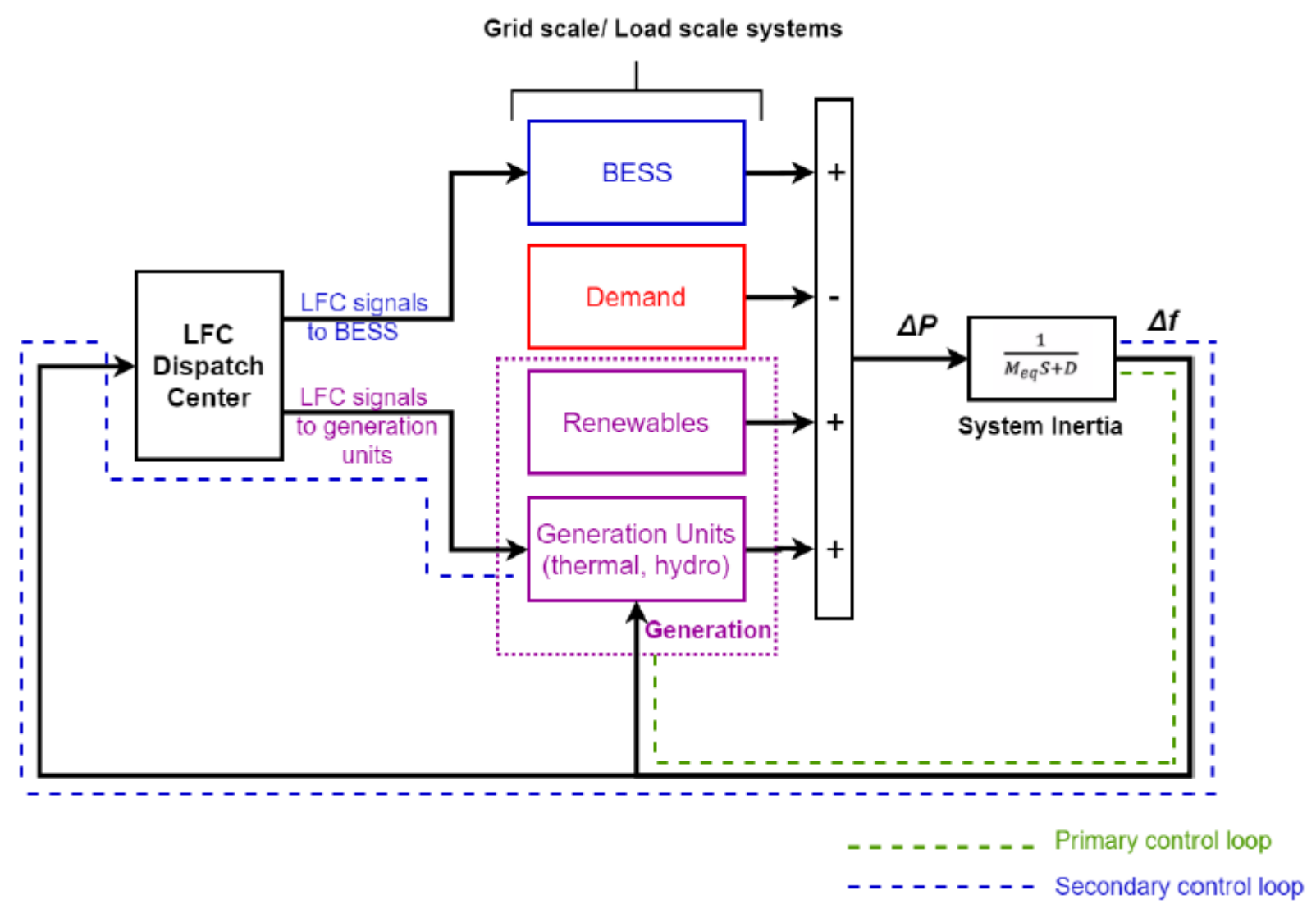

Fig. 3. A simple block diagram showing the LFC of a single area system 
As microgrids incorporate distributed BESS in their structure, this concept can inherently be adopted in multimicrogrid systems as presented in (Chowdhury and Zaman 2014). The authors studied a multi-microgrid system with two interconnected microgrids. Both ACE and frequency deviation were used in the control model to regulate the frequency of a multi-microgrid system. The situation is different in the case of isolated systems as the control signal is simply derived from only the frequency deviation of the system as the authors in (Chaitusaney and Pongthippitak 2009) presented. An isolated system consists of a wind-turbine generator, hydro plants and battery energy storage was investigated. The performance of LFC was also compared between two scenarios: With and without battery energy storage. Noticeable attenuation in system frequency fluctuation was achieved with the use BES system. However, authors in (Mohammadi et al. 2014) proposed a new LFC technique for a microgrid consists of a synchronous generator, PV unit, battery storage and loads. The contribution is to divide the microgrid into virtual areas connected via virtual tie line. So that, the LFC signals are derived from frequency deviation in addition to power deviation of a virtual tie line. Improvements in LFC performance has been reported in the results. Authors in (Hongesombut, Piroon, and Weerakamaeng 2013) have considered the effect of the power ramp rate of the thermal units on the energy capacity of the required BESS for the frequency control of a microgrid with renewable sources. A reduction of $53 \%$ in the power rate of the BESS inverter was reported.

The category of Grid Scale Systems is further reviewed by discussing the following aspects:

\section{LFC Control Strategies}

The control strategy is one of the vital research directions for LFC. As LFC is employed automatically by means of the AGC, many control strategies can be applied to implement the AGC controller. Controllers' role is to derive the control signals to the units participating in the LFC based on system requirements. We refer the interested reader to (Shayeghi, Shayanfar and Jalili 2009) for detailed information about LFC strategies. Also, the authors in (Umrao et al. 2012) have intensively categorized the controllers applied in LFC into two main groups:

- Classic control techniques: Proportional, integral, derivative and control methods based on linear quadratic regulators

- Artificial intelligence control techniques: Including fuzzy, neural, genetic, particle swarm and hybrid techniques
Each control method can be used with battery energy storage systems to propose a possible BESenhanced LFC technique. In this instance, the controller function is to issue appropriate control signals to the contributing BESS as well as the LFC participating units. Classical methods as Proportional Integral (PI), Integral (I) and Proportional-IntegralDerivative (PID) were found in (Kalyani, Nagalakshmi, and R 2012; Sen and Kumar 2014), respectively. In (L. Zhang et al. 2014), authors replaced the conventional PI controller with an H-infinity robust control method. Problems associated with parametric uncertainty and propagation delay in control channels have been considered and treated in their work. Their proposed control scheme was tested on a two area LFC of the New England 39-bus network. Results confirmed that $\mathrm{H}$-infinity controller provided better robustness and stability compared to a PI controller.

Although it is classified under "load scale systems" in our categorization, it is worth pointing out here the work in (Janfeshan, Masoum, and Deilami 2014). The authors have introduced a decentralized fuzzy logic-based control method utilizing the BES of electric vehicles. The concept of exploiting electric vehicles in frequency regulation has been discussed more deeply in the next sections.

\section{BESS Characteristics}

A battery energy storage system consists of three main modules (Anderson and Carr 1993):

- Battery pack: A set of battery cells connected in series/parallel configuration

- Power conditioning module: Power electronics converter used to interface the BES to the grid

- Control and protection module: Monitors battery state of charge SOC to ensure that the battery has the required power for its application, controls the battery charging and discharging in response to system's control variables and provides protection and safety functions

Different types of batteries can be used in BES systems for power system applications. In (Divya and Østergaard 2009), authors have listed different types of batteries with their advantages and disadvantages in different applications. Characteristics of the battery such as battery efficiency, self-discharge rate, SOC limitation, lifetime restrictions and charging and discharging rates are different for different types of batteries and play a fundamental role in the performance of LFC (Subburaj Subburaj and Bayne 2014). In general, secondary batteries used in power system applications fall into two categories:

- Conventional batteries: This includes lead-acid, Nickel-Cadmium, Sodium Sulphur, Lithium-ion

- Flow batteries: Represented by vanadium redox battery 
Sodium Sulphur is the mature battery technology that is vastly used in the grid applications (Stock et al., 2015). Figure 4 shows the estimation of the total capacity of different battery storage chemistries installed worldwide for power system applications. Lithium-ion is fast growing, and it is expected to be dominant in the near future. However, they still face high production cost compared to other batteries. One of the reasons of the high cost of Lithium-ion packs is that they require advanced protection circuitry as they are dangerously vulnerable to overcharge and overheating conditions. The protection system increases both the cost of Lithium-ion packs by $25 \%$ and their weight by $50 \%$ (Bullis 2015). NiMH batteries are still one of the popular battery technologies used in electric vehicle industries. Therefore, this battery technology can be another desirable option due to their competitive advantages of safety and low cost as well as high energy density and long life cycle (Ruegg and Thomas 2008). Presently, the creator of NiMH technology, the German and world leader of chemical industry BASF (Tullo, 2015), is researching a new NiMH technology to increase the energy capacity of NiMH batteries eightfold and reduce the price of the $\mathrm{kWh}$ to $\$ 146$ (Bullis 2015; "Nickel Metal-Hydride" 2016).

Both categories mentioned above are found in the application of LFC. Use of Sodium-Sulfur (NaS) battery system was introduced in (Shibasaki, Toge, and Iwamoto 2012). A control method based on H-infinity controller with Kalman filter for system parameters estimation has been developed. The effectiveness of NaS battery system in compensating generation fluctuations was confirmed on a two area system. Flow batteries have been distinguished among rechargeable batteries in utility applications. High power capabilities, durability, no self-discharge and fast response time of flow batteries make them an outstanding solution for LFC improvement. However, there are certain disadvantages in using redox batteries such as high cost, complexity, and low energy density compared with conventional batteries (Sasaki, Kadoya, and Enomoto 2004; Divya and Østergaard 2009). Utilization of redox flow batteries in load frequency control was studied in (Sasaki, Kadoya, and Enomoto 2004). Authors concentrated on the effectiveness of RF batteries and their promising advantages by dynamic simulation of two area interconnected system. Each system consists of thermal and hydroelectric generation units in addition to $\mathrm{RF}$ batteries. The difference of the response speed between $\mathrm{RF}$ batteries and thermal units were considered in dispatching the LFC signal. Simulation results verified the effectiveness of RF batteries features of very fast response and overload-ability in LFC application. These advantages of RF batteries were also availed of in (Ravi Shankar, Chatterjee, and Bhushan 2016) for LFC enhancement of a deregulated system.

In the aforementioned works, authors have technically discussed LFC systems utilizing one type of battery. But, effects of different characteristics of various sets of BESS have not been discussed. In (Hashimoto and Yokoyama, 2014), utilization of different sets of BESS has been investigated. The authors have developed new SOC-based control methods taking into account the different characteristics (charge and discharge rate) of two different kinds of batteries. Two sets of Nickel-Metal Hydride (NiMH) and two sets of NaS batteries each with a different capacity were considered and investigated for this purpose. Simulation results showed that the introduced control methods have appropriately improved the LFC system performance despite the difference in the charging and discharging rates of the used batteries.

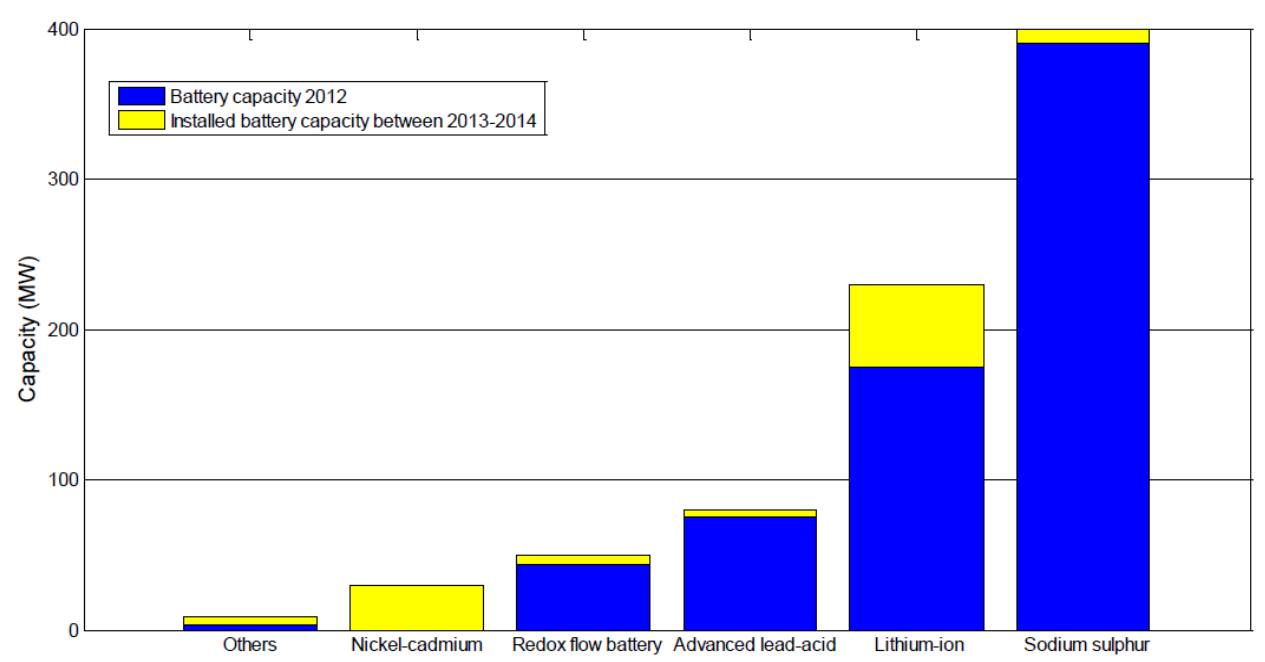

Fig. 4. The estimation of the total battery capacity installed worldwide for power system applications (reproduced with permission from (Stock et al., 2015)) 


\section{Optimal Sizing of BESS}

One of the main challenges encounters the deployment of BES in utility applications is the cost of BES systems ("Grid Energy Storage" 2013). Authors in (Cook, Spindler, and Grefe 1991) stated that "before one can discuss the cost of BES, it is necessary to define the size of it." Therefore, optimization of BES size must be considered to achieve the highest profitability for the application (A. Oudalov et al. 2006; Alexandre Oudalov, Cherkaoui, and Beguin 2007). On the other hand, the size of batteries is foremost selected to meet the power and energy requirements of the LFC. Thus optimal sizing of BES should be achieved. Different research works have been reported on this topic (Mercier, Cherkaoui, and Oudalov 2009). The significant matter that needs to be tackled in deciding the battery size is the difference in response speed between batteries and other LFC participating units. In (Amano et al. 2012), authors presented a new method for ACE assignment to the BESS and LFC units in order to curtail the required capacity of BESS. The proposed method in (Amano et al. 2012) basically assigns low-frequency components of ACE signal to slow LFC generation units while highfrequency components are dispatched to faster BESS. A similar approach has been discussed in (Leitermann and Kirtley 2010) to reduce the ramp rate requirement of the thermal units and the required capacity of BES units. A strategy of using BESS in LFC considering reduction of BES capacity has also been presented in (Nagoya, Komami, and Ogimoto 2013). Authors discussed the issue of the difference in speed of response of BESS and thermal units by dividing the duty of LFC between them. BESS take care of frequency regulation function while load-following is assigned to thermal units. So that, fast BESS are involved in short periods of charge and discharge and do not suppress thermal units. Consequently, reduction in energy capacity of BES can be achieved. The contribution is to derive LFC signals of BESS from deviation in tie line power. Otherwise, LFC signals to the thermal units are derived from deviation in tie line power in addition to battery power output. This can satisfy the situation where the output of thermal units does not depend on BESS. As a result, 30\% reduction in BES capacity was achieved. In (Mercier, Cherkaoui, and Oudalov 2009), authors developed a strategy to optimize the size of BESS to maximize the operating profit using Net Present Value (NPV) formula.

\section{Summary of Grid-Scale Systems}

All the work reviewed under this category focused on grid-tied BES systems in large scales and used in coordination with generation-side control. This technology is being vastly studied in power systems.
However, it encounters practical challenges as summarized below:

- The high cost of installation of large capacity BES systems

- BES systems with high efficiency are required

- Requirements for the coordinated dispatch of LFC signals between thermal generation units, BES units and system operator because of time scale difference between BESS and generation units

Recently, more attention is being focused on demand-side control using customers' loads to participate in frequency regulation and share the burden with generation-side control. Therefore, loads equipped with battery energy storage can also effectively contribute to reducing the requirements of BES capacity (Zhao, Topcu, and Low 2012). In the next section, techniques of LFC with BES using local loads as "controlled reserve" are highlighted.

\section{Load-Scale Systems}

Frequency deviation in the power system can be instantaneously sensed at any point in the generation, transmission and distribution systems. It can also be measured locally at home outlets as a system-wide indicator of load-generation balance (Ota et al. 2010). Therefore, loads can play a significant role to actively control system frequency instead of being passive elements sitting on the grid. Before we discuss the LFC techniques under this category, first we briefly introduce the concept of using loads in frequency regulation i.e. demand side control. Recently, many research articles have been carried out in this domain (Zhao Xu, Østergaard, and Togeby 2011; Z. Xu et al. 2007; Biegel et al. 2013). Loads such as heat pump water heaters, resistive heaters and refrigerators are considered suitable candidates for this application due to their capability of thermal energy storage (Douglass et al. 2013). In (Taisuke Masuta, Yokoyama, and Tada 2010; Kinjyo et al. 2012; T. Masuta, Yokoyama, and Tada 2011), thermal energy storage using heat pump water heaters was utilized for LFC support. Such loads can be controlled (switched $\mathrm{ON}$ or OFF) to track frequency fluctuations momentarily. Authors in (Zhao, Topcu, and Low 2012) have listed a number of research works that have been conducted about frequency-based load control strategy. Among them is the Grid Friendly Controller of the Pacific Northwest National Laboratory ("Grid Friendly ${ }^{\mathrm{TM}}$ Controller Helps Balance Energy Supply and Demand" 2012). This controller senses the line frequency and controls appliances accordingly. When the frequency is below scheduled value $(60 \mathrm{~Hz}$ in U.S.), the controller switches the appliance off for a period of few seconds to few minutes in order to reduce the load. 
Though feasible, this frequency-based load control has some drawbacks of "end-user disutility" because appliances will be turned off during overload periods. Disutility periods must be minimized in order to lessen users inconvenience, or this strategy might not be accepted by customers (Zhao, Topcu, and Low 2012). However, in (Zhao, Topcu, and Low 2013), an approach of decentralized load control based on frequency monitoring has been presented to minimize the disutility of loads. Communication between loads was needed in order to alleviate the effect of inaccuracies in frequency measurement at different loads points. Therefore, a tradeoff between shortest disutility periods and simplest communication must be made. Another challenge of such a control strategy is in providing the regulation during the over-frequency condition. In this posture, a group of appliances should be dedicated and scheduled to be turned $\mathrm{ON}$ when the frequency exceeds the rated value. Therefore, a scheduling algorithm is required to manage the appliances ON/OFF switching not to interfere with users' convenience. This issue has been discussed in (Biegel et al. 2013) by proposing an ON/OFF strategy for the appliances to enable primary reserve in both directions and considering three levels of user convenience, namely "optimistic," "conservative," and "probabilistic." A new concept of demand response referred to as "electric spring" has been proposed and developed by the authors of the works in (Lee, Cheng, and $\mathrm{Ng}$ 2013; Tan, Lee, and Hui 2013; Hui, Lee, and $\mathrm{Wu}, 2012$ ). The authors of the electric spring system used power electronics technology to implement an innovative idea of "smart loads".

The aforementioned control strategies are beyond the scope of our review as we specifically discuss the enlistment of battery energy storage in frequency regulation. Therefore, we will focus on the demand side response with BESS.

Customers loads equipped with battery energy storage can be used for LFC enhancement. This is defined as the category of load-scale systems in our classification. In this category, distributed battery energy storages are used at the load side as auxiliary storages and controlled in a manner analogous to the AGC of thermal generation units based on locally available data. Currently, the solely used and widely discussed appliance for this application is electric vehicles (EVs). Different types of electric vehicles (plug-in hybrid electric vehicles and "All-Electric" vehicles as classified by the U.S. DoE ("Hybrid and Plug-In Electric Vehicles" 2015)) can be applied for LFC as mobile BES units (Shimizu et al. 2010; Galus, Koch, and Anderson 2011). According to the way of cooperation between controlled loads and power system operators, the strategy of loadscale systems can be classified into two groups: Coordinated Aggregate Systems and Non-Coordinated Individual Systems.

\section{Coordinated Aggregate Systems}

The category of aggregate systems has been found dominant among the existing load-scale systems. EVs are the pioneering realistic appliances that are being investigated to be used in this strategy. However, other methods of distributed battery storage such as other battery-equipped appliances or domestic battery banks can be feasibly used ("Tesla Power Wall/ Home Battery" 2016; Bila, Opathella, and Venkatesh 2016; Adika and Wang 2014). In this category, a number of EVs (in V2G operation mode) is connected to a centralized charging station with a high level of coordination between EVs and the system operator through two-way communication. An intermediate controller and administrator defined (using the term in (Guille and Gross 2009)) as "the aggregator" gathers information of battery SOC, plug-in and plug-out time and predicted arrival and next charging time from smart interfaces of each EV and communicate with the dispatching center. Then LFC signals are dispatched to the EVs via the aggregator (Kennel, Görges, and Liu 2013). Authors in (Shimizu et al. 2010) discussed an LFC scheme utilizing the BES of electric vehicles as a means to reduce the overall requirement of BESS. A control method takes into consideration the SOC of the battery of EVs was developed giving priority to users. The suggested SOC control strategy controls only the plugged EVs that are fully charged (SOC $=80-90 \%$ ) in order not to affect users' convenience. The studied system consists of 50000 EVs distributed on 500 local control centers that are communicating with a central load dispatching center. The system was investigated under two scenarios that are considering user convenience and not considering user convenience. Results showed that the frequency response is better without taking into account user convenience than when considering user convenience because, in the later case, the output of EVs is restricted by SOC limits. Provision of LFC utilizing the BESS of distributed Plug-in Hybrid Electric Vehicles (PHEV) along with thermostatically controlled loads has been proposed in (Galus, Koch, and Anderson 2011). Authors developed an aggregation method implemented through a management system defined as the aggregator based on model predictive control method. The developed aggregator ensures a high level of coordination between PHEV and the controllable loads, distribution network and system operators via communication. The studied system consists of a cluster of $40000 \mathrm{PHEV}$, groups of thermostatically controlled appliances (cooling and heating appliances) and a CHP unit all connected to a network serving an urban community of 160,000 inhabitants. It was shown by the results that such frequency controlled reserves could effectively be used to enhance LFC of the system. However, authors addressed a significant question about the sufficiency and availability of the reserve amount 
provided by such loads in the practical implementation of the proposed scheme for further scheme. Furthermore, the effects of delay in communication systems were not discussed and the aggregation effectiveness has been presented under the condition of very fast communication. The impact of communication and data exchange technology between LFC-controlled loads and the grid has been discussed in (Wada and Yokoyama 2012). Via numerical simulation, authors considered the network delay (on the network) and processing time delay (on the server) to dispatch LFC signals to the distributed batteries at the demand side. However, network delay was also neglected. The system was studied under large penetration of wind energy, up to $1800 \mathrm{MW}$ and random load fluctuation of average 8000 MW. Three different communication models (processing delay) and different installation rates of the battery storage were discussed. A control scheme called "cutoff control" was slow developed to cope with slow response batteries. A challenge can be highlighted in this method which is the low utilization of the BESS as the slow batteries are not dispatched in certain operating conditions. Authors have also addressed another challenge that is related to considering SOC limits in the simulation time to avoid battery over-charge or over-discharge.

The issue of SOC limits was tackled in (Kennel, Görges, and Liu 2013) by developing an energy management system using Hierarchical Model Predictive Control (HiMPC) to support frequency regulation of a smart grid of a city. The studied system includes intermittent renewable energy sources as well as conventional generators. Moreover, the system incorporates coordinated electric vehicles. The SOC of each electric vehicle is predicted and managed by the proposed HiMPC to avoid over-discharge and over-charge states. Significant improvement in LFC performance was reported using electric vehicles compared to the case when electric vehicles are not available for control.

In (Vachirasricirikul and Ngamroo 2014), authors proposed a new control strategy of $\mathrm{V} 2 \mathrm{G}$ scheme for frequency support. The motivation is to cope with the difficulties related to system disturbances and uncertainties and to achieve a robust controller with a simple structure. Both classical and intelligent control methods were used where a particle swarm optimization method based on mixed $\mathrm{H} 2 / \mathrm{H}$-infinity control was used to adjust the parameters of the frequency controller. The frequency controller is simply implemented with a conventional PI controller. The power output of the battery is controlled based on SOC optimization technique to maintain battery SOC within a predefined range. The effectiveness and robustness of the introduced control method were verified by simulation results of a two-area system incorporating a large wind farm.
The effectiveness of using EVs in LFC was also studied in (Huang et al. 2012). The list of publications about the contribution of EVs in frequency regulation is fairly long, but we aim to highlight the importance of LFC control and the audience can find more information in the literature about the vehicle to grid technology.

Based on the previous discussion, the following challenges in this category must be tackled:

- The charge and discharge control must prioritize the customer requirements and convenience

- To achieve the above service, system operators must be able to monitor and manage a mass of individual loads. Therefore, intermediary aggregation and management entities are needed to dispatch LFC signals locally and in coordination with the generation side

- With the absence of knowledge of the entire system status, the aggregator evaluates the necessary requirements of LFC services from local operating conditions. This needs to be achieved by metering, collection and management of real-time information of loads statuses such as line frequency, battery SOC limits and, in the case of EVs, plug-in and plug-out times (Mullen 2009)

- The coordination between aggregators and utility operators necessitates reliable two-way communication infrastructure with sufficiently fast protocols (S. Liu, Wang, and Liu 2015)

The abovementioned requirements add more installation and operational costs to the system, Therefore; it is essential to achieve optimal tradeoffs between cost, control complexity, the amount of communication and benefits of frequency regulation in order for the utility operators to accept this load control strategy.

\section{Non-Coordinated Individual Systems/Primary Frequency Control}

With advance developments in power electronics and control strategies, battery-powered appliances can be equipped with interface circuits that have the ability to precisely measure system frequency and respond to power fluctuations (Ribeiro et al. 2001; Ota et al. 2010). The concept of non-coordinated control of individual battery-equipped loads to achieve demand-side frequency regulation is a field of study that is still on the drawing board. As in the coordinated aggregate systems, EVs are found the dominant candidate where decentralized control is achieved at EV's end via vehicle chargers. Therefore, smart interface circuits that are capable of frequency measurement and realizing effective control of bidirectional power flow between the battery and power line based on the measured frequency condition are required. In (Rei et al. 2010), 
the authors developed an efficient and smart power interface circuit for EVs. The circuit is based on a three-phase multilevel diode clamped topology as a bidirectional AC/DC converter. This circuit implements ancillary frequency and voltage regulation services according to the droop control strategy. Based on a similar idea, a single phase "Grid-Friendly" interface circuit was developed in (Ota et al. 2012) to realize the smart charging and $\mathrm{V} 2 \mathrm{G}$ concepts.

The motivation behind the non-coordinated individual systems is to dispense the requirements of communication and cyber security between controllable loads and system operators for a more flexible system as argued (Molina-Garcia, Bouffard, and Kirschen 2011). Basically, this approach is similar to the idea of frequency-based load control and Grid Friendly Controller discussed earlier (Donnelly et al., 2008). Nevertheless, instead of switching off the appliance during under-frequency periods, the charge-discharge process of load batteries is managed with a droop control strategy corresponding to generators droop control. Therefore, this concept no longer falls in the secondary control category by definition. One way or another, it realizes the primary frequency control as no signal of coordination between the battery and the generation side is sent. The line frequency signal embodies the only communication signal between the load battery and the generation side.

The Smart Frequency-Sensing Charge Controller for Electric Vehicles developed by Argonne National Laboratory proposes that battery loads of PEV can be automatically controlled in response to line frequency using the droop control and without any signal from the dispatching center (Koritarov 2013). The controller reduces the charging rate to $50 \%$ when the frequency deviation is in range $0.1 \mathrm{~Hz}<\Delta \mathrm{f}<0.5 \mathrm{~Hz}$ whereas it completely stops charging (shedding the charging load) when the frequency drops below $59.5 \mathrm{~Hz}$. The charging state with $50 \%$ rate is resumed when the power mismatch is healed and frequency is greater than $59.7 \%$. Full-rate charging is applied when the frequency is greater than $60 \mathrm{~Hz}$. This regulation strategy is defined as smart charging or V1G where one-way power transfer (grid-to-vehicle) is allowed and no power is discharged during under-frequency periods to support the utility as in the V2G strategy.

Authors in (Ota et al. 2010) proposed a noncoordinated and distributed V2G scheme through twoway power transfer between the vehicle and the grid. However, V1G mode is also adopted to maintain the SOC of the vehicle battery within the range required by users. The presented control strategy suggests that the battery power is modulated in proportional to frequency deviation as droop characteristics with SOC balancing algorithm. The SOC balancing algorithm ensures safe charge/discharge of the battery and sustains its SOC between 10-90\%. Maximum (charge/discharge) power is achieved at frequency deviation of $(+0.025 \mathrm{~Hz} /-0.025$ $\mathrm{Hz}$ ) respectively. Upon driver request, the control is switched between V2G and V1G modes. The effect of a total of $250 \mathrm{MW} \mathrm{V2G}$ power was investigated in a twoarea thermal system without any coordination between the vehicles and the thermal generators or the system operator. Obvious improvement in the frequency control of both areas was achieved keeping the SOC in its predefined boundary around $50 \%$ level. For the user to hold its battery SOC around a different level, the SOC parameters in the balancing algorithm need to be modified. Therefore, a SOC holding algorithm was developed in (H. Liu et al. 2013) which maintains the SOC level at the user preferred setting without changing the SOC boundary limits.

As it was mentioned earlier, EVs are the widely discussed battery-equipped appliance for this control strategy. However, recently, more attention has been focused on household batteries for demand-side control in the emerging technology of Behind-the-Meter Energy Storage coinciding with the release of advanced home batteries by battery industry leaders such as the "Powerwall" of Tesla and "xStorage" of Nissan earlier this year. However, based on a study conducted by the Rocky Mountain Institute (Fitzgerald et al. 2015), behindthe-meter storage faces different challenges that keep it from providing frequency regulation services in addition to the end user-oriented services. The study listed these challenges, the steps to avoid them and the entities that should take each of the suggested steps to enable this paradigm of battery storage to serve in the frequency regulation. The research community is one of the relevant entities, based on the study and it is required to invest efforts in developing technical and economic methodologies in this focus. Through this survey, different attempts were found in the literature. Performance analysis of a household-size lithium-ion battery energy storage was presented by (Bila, Opathella and Venkatesh 2016). The cycling capabilities and energy efficiency of the considered battery energy storage were evaluated from experimental measurements. Decentralized control algorithms of groups of household batteries were discussed by (Adika and Wang 2014) and (Tao and Chen 2015) based on the game theory. (Kim, Del-Rosario-Calaf, and Norford 2016) discussed the use of PEV as behind-the-meter energy storage units in a new control strategy for both primary and secondary frequency control.

The non-coordinated individual systems aim to prioritize users' convenience from the point of view of SOC and to protect their privacy by reducing communications with the system operator. 


\section{Conclusion}

Battery energy storage is found essential for increasing the quality of the load frequency control in power systems with a high amount of renewables. This approach can be implemented with large capacity BESS operated by the utility in accordance with load frequency controller. Such a topology requires coordinated dispatch of LFC signal between generation units, BESS and system operator considering the time scale difference between BESS and the generation units. With much attention is being given to demand side control, the approach of using BESS in LFC can also be implemented using distributed batteries embedded in loads or sited at customers' homes to reduce the required the capacity of the centralized storage. EVs are extensively discussed as a feasible candidate to be enlisted as distributed auxiliary storages. But, accompanied by the recent development of advanced home batteries such as Tesla's Powerwall and its rivals represented by Nissan's xStorage, Orison's home battery and Mercedes-Benz's home battery, EVs are not the only possible battery storages to be used in frequency regulation. In this context, system operators need to monitor and manage a bulk of individual loads. Therefore, intermediary aggregation and management entities are required to dispatch LFC signals locally and in coordination with the generation entity. The aggregator evaluates the necessary requirements of LFC services and users' convenience from local operating conditions through metering and management of realtime information of loads conditions such as line frequency, battery SOC limits and plug-in and plug-out times. Therefore, the coordination between aggregators and utility operators necessitates reliable two-way communication infrastructure with fast protocols to respond within the LFC time window. Advanced power electronics and control strategies enable the management systems of end-users' batteries to be equipped with smart circuits to measure the line frequency at home sockets and adjust battery charge and discharge activities accordingly. This has inspired researchers to investigate autonomous and distributed initiatives for primary frequency control to reduce the coordination with system operators and eventually remove communication requirements toward decentralization. However, some degrees of coordination between the loads batteries on one hand and between the batteries and the system operator, on the other hand, are still required to achieve pooling management. Pooling management realizes SOC synchronization and participation scheduling to ensure that the aggregated power provided by the batteries is sufficiently high enough for the required frequency control services. By eliminating the communication with the system operator, autonomous systems do not provide LFC services.
Instead, they serve as primary control reserves with droop control characteristics using the line frequency as the only negotiation signal with the operators. This emerging scheme requires practical implementations to prove its concept and evaluate its effectiveness for utilities. With the emergence of the behind-the-meter energy storage technology for customer side services, frequency regulation can also be one of the feasible and profitable services offered by this technology.

\section{Author's Contributions}

Both authors contributed to the preparation of this work.

\section{Ethics}

The authors have no conflict of interest to reveal.

\section{References}

Adika, Christopher O., and Lingfeng Wang. 2014. "Non-Cooperative Decentralized Charging of Homogeneous Households' Batteries in a Smart Grid." IEEE Transactions on Smart Grid 5 (4): 1855-63. doi:10.1109/TSG.2014.2302449.

Aditya, S.K, and D Das. 2001. "Battery Energy Storage for Load Frequency Control of an Interconnected Power System." Electric Power Systems Research 58 (3): 179-85. doi:10.1016/S0378-7796(01)00129-8.

Aditya, S K, and D Das. 1999. "Application of Battery Energy Storage System to Load Frequency Control of an Isolated Power." International Lournal of Energy Research 258 (July 1998): 247-58.

Agrawal, Aishvarya, Mahesh Kumar, Dharmendra Kumar Prajapati, Mukesh Singh, and Praveen Kumar. 2014. "Smart Public Transit System Using an Energy Storage System and Its Coordination with a Distribution Grid." IEEE Transactions on Intelligent Transportation Systems 15 (4): 1622-32.

Akhil, Abbas. 1995. "Trends and Status of Battery Energy Storage for Utility Applications." In Proceedings of the Tenth Annual Battery Conference on Applications and Advances, 273-77.

Alam, M. J. E., Kashem M. Muttaqi, and Danny Sutanto. 2013. "A Controllable Local Peak Shaving Strategy for Effective Utilization of PEV Battery Capacity for Distribution Network Support." IEEE Transactions on Industry Applications. doi:10.1109/TIA.2014.2369823.

Amano, Hiroyuki, Yuji Ohshiro, Tomonori Kawakami, and Toshio Inoue. 2012. "Utilization of Battery Energy Storage System for Load Frequency Control toward Large-Scale Renewable Energy Penetration." In Innovative Smart Grid Technologies (ISGT Europe), 2012 3rd IEEE PES International Conference and Exhibition on, 1-7. 
Anderson, M A X D, and Dodd S Carr. 1993. "Battery Energy Storage Technologies." Proceedings of the IEEE 81 (3): 475-79.

Bakken, Bjorn H, and Ove S Grande. 1998. "Automatic Generation Control in a Deregulated Power System." IEEE Transactions on Power Systems 13 (4): 1401-6.

"Balancing and frequency control: A Technical Document Prepared by the NERC Resources Subcommittee," 2011. [Online]. Available: http://www.nerc.com/docs/oc/rs/NERC. [Accessed: 07-Aug-2014].

Beiter, Philipp. 2014. “2014 Renewable Energy Data Book."

Bhatt, Ravi, and Badrul Chowdhury. 2011. "Grid Frequency and Voltage Support Using PV Systems with Energy Storage." In North American Power Symposium (NAPS), 2011, 1-6.

Biegel, Benjamin, Lars Henrik Hansen, Palle Andersen, and Jakob Stoustrup. 2013. "Primary Control by ON/OFF Demand-Side Devices." IEEE Transactions on Smart Grid 4 (4): 2061-71. doi:10.1109/TSG.2013.2257892.

Bila, M., C. Opathella, and B. Venkatesh. 2016. "Grid Connected Performance of a Household Lithium-Ion Battery Energy Storage System." Journal of Energy Storage 6. Elsevier Ltd: 178-85. doi:10.1016/j.est.2016.04.001.

Bullis, Kevin. 2015. "Old Battery Type Gets an Energy Boost," MIT Energy Review, 2015. [Online]. Available:

https://www.technologyreview.com/s/535251/oldbattery-type-gets-an-energy-boost/. [Accessed: 06Jun-2016].

Cha, Seung Tae, Haoran Zhao, Qiuwei Wu, Arshad Saleem, and Jacob Ostergaard. 2012. "Coordinated Control Scheme of Battery Energy Storage System (BESS) and Distributed Generations (DGs) for Electric Distribution Grid Operation." IECON 2012 - 38th Annual Conference on IEEE Industrial Electronics Society, October. Ieee, 4758-64. doi:10.1109/IECON.2012.6389587.

Chaitusaney, S., and P. Pongthippitak. 2009. "Integration of Battery for Attenuating Frequency Fluctuation due to Wind Turbine Generation." In Electrical Engineering/Electronics, Computer, Telecommunications and Information Technology, 2009. ECTI-CON 2009. 6th International Conference on.

Chowdhury, A Hasib, and Md. Asaduz Zaman. 2014. "Load Frequency Control of Multi-Microgrid Using Energy Storage System." In 8th International Conference on Electrical and Computer Engineering, 548-51.

Cook, G. M., W. C. Spindler, and C. Grefe. 1991. "Overview of Battery Power Regulation and Storage." IEEE Transaction on Energy Conversion 6 (1): 204-11.
Divya, K.C., and Jacob Østergaard. 2009. "Battery Energy Storage Technology for Power systems-An Overview." Electric Power Systems Research 79 (4): 511-20. doi:10.1016/j.epsr.2008.09.017.

Dong, D., P. Wang, W. Qin, and X. Han. 2014. "Investigation of a Microgrid with Vanadium Redox Flow Battery Storages as a Black Start Source for Power System Restoration." In Proceedings of the 2014 9th IEEE Conference on Industrial Electronics and Applications, ICIEA 2014, 140-45. doi:10.1109/ICIEA.2014.6931147.

Donnelly, Matthew K., David P. Chassin, Jeffery E. Dagle, Michael Kintner-Meyer, David W. Winiarski, Robert G. Pratt, and Anne Marie Boberly-Bartis. 2008. Electrical appliance energy consumption control methods and electrical energy consumption systems. US7420293 B2, issued 2008.

Douglass, Philip J, Rodrigo Garcia-valle, Preben Nyeng, Jacob Østergaard, and Mikael Togeby. 2013. "Smart Demand for Frequency Regulation: Experimental Results." IEEE Transaction on Smart Grid 4 (3): 1713-20.

Fadrhonc, W, J Matamoros, and P Sood. 2014. "Enlisting Conventional Power Electronic Devices to Improve Stability and Security through Distributed Load Shedding and Energy Storage." In 2014 IEEE PES T\&D Conference and Exposition.

Fitzgerald, Garrett, James Mandel, Jesse Morris, and Hervé Touati. 2015. "The Economics of Battery Energy Storage."

Galus, Matthias D, Stephan Koch, and Goran Anderson. 2011. "Provision of Load Frequency Control by PHEVs, Controllable Loads, and a Cogeneration Unit." IEEE Transaction on Industrial Electronics 58 (10): 4568-82.

Ghiassi-farrokhfal, Yashar, Srinivasan Keshav, and Catherine Rosenberg. 2015. "Toward a Realistic Performance Analysis of Storage Systems in Smart Grids." IEEE Transaction on Smart Grid 6 (1): 402-10.

Goldsbrouch, W.E., and P. E. Fansler. 1903. "The Storage-Battery in Substations." In The 20th Annnal Conivention of the American Institute of Electrial Engineers.

“Grid Energy Storage." 2013. U.S. Department of Energy. http://energy.gov/oe/downloads/gridenergy-storage-december-2013.

"Grid Friendly TM Controller Helps Balance Energy Supply and Demand." 2012.

Guille, Christophe, and George Gross. 2009. "A Conceptual Framework for the Vehicle-to-Grid (V2G) Implementation." Energy Policy 37 (11): 4379-4390. 
Hashimoto, Atsushi, and Akihiko Yokoyama. 2014. "A New Control Method of Multiple Sets of Battery Energy Storage System for Load Frequency Control in Power System with Massive Integration of Renewable Energy Resources.” In Power Systems Computation Conference (PSCC).

Hongesombut, K., T. Piroon, and Y. Weerakamaeng. 2013. "Evaluation of Battery Energy Storage System for Frequency Control in Microgrid System.” In 2013 10th International Conference on Electrical Engineering/Electronics, Computer, Telecommunications and Information Technology, ECTI-CON 2013, 3-6. doi:10.1109/ECTICon.2013.6559493.

Huang, Yuan, Jun-yong Liu, Cheng-xin Li, and Wei Gong. 2012. "Considering the Electric Vehicles in the Load Frequency Control." In 2012 Asia-Pacific Power and Energy Engineering Conference, 0-3.

Hui, Shu Yuen, Chi Kwan Lee, and Felix F. Wu. 2012. "Electric Springs - a New Smart Grid Technology." IEEE Transactions on Smart Grid 3 (3): 1552-61. doi:10.1109/TSG.2012.2200701.

"Hybrid and Plug-In Electric Vehicles." 2015. U.S. Department of Energy - Energy Efficiency and Renewable Energy/Alternative Fuels Data Center. Accessed March http://www.afdc.energy.gov/vehicles/electric.html.

Jaleeli, Naseer, Louis S. VanSlyck, Donald N. Ewart, Lester H. Fink, and Arthur G. Hoffmann. 1992. "Understanding Automatic Generation Control." IEEE Transactions on Power Systems 7 (3): 1106-22.

Janfeshan, Keyhaneh, Mohammad a.S. Masoum, and Sara Deilami. 2014. "V2G Application to Frequency Regulation in a Microgrid Using Decentralized Fuzzy Controller." In Proceedings of 2014 International Conference on Modelling, Identification \& Control, 361-64. Ieee. doi:10.1109/ICMIC.2014.7020781.

Joseph, Ami, and Mohammad Shahidehpour. 2006. "Battery Storage Systems in Electric Power Systems." In 2006 IEEE Power Engineering Society General Meeting, 1-8.

Jung, Kyung-Hee, Hoyong Kim, and Daeseok Rho. 1996. "Determination of the Instalation Site and Optimal Capacity of the Battery Energy Storage for Load Leveling." IEEE Transactions on Energy Conversion 11 (1): 162-67.

Kalyani, S., S Nagalakshmi, and Marisha. R. 2012. "Load Frequency Control Using Battery Energy Storage System in Interconnected Power System." In Computing Communication \& Networking Technologies (ICCCNT), 2012 Third International Conference on.
Kennel, Fabian, Daniel Görges, and Steven Liu. 2013. "Energy Management for Smart Grids with Electric Vehicles Based on Hierarchical MPC." IEEE TRANSACTIONS ON INDUSTRIAL INFORMATICS 9 (3): 1528-37.

Khalid, Muhammad, and Andrey V. Savkin. 2010. "Model Predictive Control Based Efficient Operation of Battery Energy Storage System for Primary Frequency Control." In 2010 11th International Conference on Control Automation Robotics \& Vision, 2248-52. Ieee. doi:10.1109/ICARCV.2010.5707855.

Kim, Young-Jin, Gerard Del-Rosario-Calaf, and Leslie $\mathrm{K}$ Norford. 2016. "Analysis and Experimental Implementation of Grid Frequency Regulation Using Behind-the-Meter Batteries Compensating for Fast Load Demand Variations." IEEE Transaction on Power Systems 8950 doi:10.1109/TPWRS.2016.2561258.

Kinjyo, Yoshihisa, Masaya Miyagi, Tomonobu Senjyu, Atsushi Yona, and Toshihisa Funabashi. 2012. "Decentralized Controllable Loads Control in Small Power System.” In 2012 International Conference on Renewable Energy Research and Applications (ICRERA), 1-6. Ieee. doi:10.1109/ICRERA.2012.6477475.

Koritarov, Vladimir. 2013. Frequency based electric vehicle charge controller system and method for implementing demand response and regulation services to power grid using frequency detection. US20130033234 A1, issued 2013.

Kottick, D., M. Blau, and D Edelstein. 1993. "Battery Energy Storage for Frequency Regulation in an Island Power System." IEEE Transaction on Energy Conversion 8 (3): 455-59.

Kundur, Prabha. 1993. Power System Stability And Control. Edited by Neal J. Balu and Mark G. Lauby. New York: McGraw-Hill, Inc.

Kunisch, H.-J., K.G. Kramer, and H Dominik. 1986. "Battery Energy Storage Another Option for LoadFrequency-Control and Instantaneous Reserve." IEEE Transactions on Energy Conversion EC-1 (3): 41-46.

Lee, Chi Kwan, Kai Lok Cheng, and Wai Man Ng. 2013. "Load Characterisation of Electric Spring." In 2013 IEEE Energy Conversion Congress and Exposition, ECCE 2013, 4665-70. doi:10.1109/ECCE.2013.6647326.

Leitermann, Olivia, and James L Kirtley. 2010. "Energy Storage for Use in Load Frequency Control." In Innovative Technologies for an Efficient and Reliable Electricity Supply (CITRES), 2010 IEEE Conference on, 292-96. 
Li, Xinran, Yawei Huang, Jiyuan Huang, Shaojie Tan, Ming Wang, Tingting $\mathrm{Xu}$, and Xingting Cheng. 2014. "Modeling and Control Strategy of Battery Energy Storage System for Primary Frequency Regulation." In 2014 International Conference on Power System Technology, 543-49. Ieee. doi:10.1109/POWERCON.2014.6993760.

Lian, Bo, Dongmin Yu, Cheng Wang, Simon Le Blond, and Roderick W Dunn. 2014. "Investigation of Energy Storage and Open Cycle Gas Turbine for Load Frequency Regulation." In Power Engineering Conference (UPEC), 2014 49th International Universities.

Liu, Hui, Zechun Hu, Yonghua Song, and Jin Lin. 2013. "Decentralized Vehicle-to-Grid Control for Primary Frequency Regulation Considering Charging Demands." IEEE Transaction on Power Systems 28 (3): 3480-89.

Liu, Shichao, Xiaoyu Wang, and Peter Xiaoping Liu. 2015. "Impact of Communication Delays on Secondary Frequency Control in an Islanded Microgrid." IEEE Transactions on Industrial Electronics 62 (4): 2021-31.

Liu, Weijia, Lei Sun, Zhenzhi Lin, Fushuan Wen, and Yusheng Xue. 2016. "Multi-Objective Restoration Optimisation of Power Systems with Battery Energy Storage Systems." IET Generation, Transmission \& Distribution 10 (7): 1749-57. doi:10.1049/ietgtd.2015.0434.

Lu, Chun-feng, Chun-Chang Liu, and Chi-Jui Wu. 1995. "Effect of Battery Energy Storage System on Load Frequency Control Considering Governor Deadband and Generation Rate Constraint." IEEE Transactions on Energy Conversion 10 (3): 555-61.

Martínez, Ernesto Vázquez, and José Antonio de la O Serna. 2015. "Smart Grids Part 1 : Instrumentation Challenges." IEEE Instrumentation \& Measurement Magazine.

Masuta, T., A. Yokoyama, and Y. Tada. 2011. "Modeling of a Number of Heat Pump Water Heaters as Control Equipment for Load Frequency Control in Power Systems." In 2011 IEEE Trondheim PowerTech, 1-7. Ieee. doi:10.1109/PTC.2011.6019457.

Masuta, Taisuke, Akihiko Yokoyama, and Yasuyuki Tada. 2010. "System Frequency Control by Heat Pump Water Heaters (HPWHs) on Customer Side Based on Statistical HPWH Model in Power System with a Large Penetration of Renewable Energy Sources." In International Conference on Power System Technology, 1-7.

Mehr, Tahoura Hosseini, Mohammad A S Masoum, and Nasim Jabalameli. 2013. "Grid-Connected LithiumIon Battery Energy Storage System for Load Leveling and Peak Shaving." In Australasian Universities Power Engineering Conference.
Mercier, Pascal, Rachid Cherkaoui, and Alexandre Oudalov. 2009. "Optimizing a Battery Energy Storage System for Frequency Control Application in an Isolated Power System." IEEE Transaction on Power Systems 24 (3): 1469-77.

Mohammadi, F Doost, M Jawad Ghorbani, A Feliachi, and M A Choudhry. 2014. "Novel Load Frequency Control Approach Based on Virtual Area Error in a Microgrid Including PV and Battery." In PES General Meeting | Conference \& Exposition, 2014 IEEE, 1-5.

Molina-Garcia, Angel, Francois Bouffard, and Daniel S. Kirschen. 2011. "Decentralized Demand-Side Contribution to Primary Frequency Control." IEEE Transactions on Power Systems 26 (1): 411-19. doi:10.1109/TPWRS.2010.2048223.

Mullen, Sara Kathryn. 2009. "Plug-In Hybrid Electric Vehicles as a Source of Distributed Frequency Regulation." Control. University of Minnesota. http://conservancy.umn.edu/bitstream/56792/1/Mull en_umn_0130E_10730.pdf.

Nagoya, Hiroyuki, Shintaro Komami, and Kazuhiko Ogimoto. 2013. "A Method for Load Frequency Control Using Battery in Power System with Highly Penetrated Photovoltaic Generation." Electrical Engineering in Japan 184 (4): 22-31. doi:10.1002/eej.22425.

"Nickel Metal-Hydride." 2016. BASF. Accessed September 9. http://www.catalysts.basf.com/p02/USWebInternet/catalysts/en/content/microsites/catalysts/pro ds-inds/batt-mats/NiMH.

Noce, C., S. Riva, G. Sapienza, and M. Brenna. 2012. "Electrical Energy Storage in Smart Grid: BlackStart Study Using a Real-Time Digital Simulator." In 2012 3rd IEEE International Symposium on Power Electronics for Distributed Generation Systems (PEDG), 216-20. doi:10.1109/PEDG.2012.6254004.

Ota, Y, H Taniguchi, T Nakajima, K M Liyanage, J Baba, and A Yokoyama. 2010. "Autonomous Distributed V2G (Vehicle-to-Grid) Considering Charging Request and Battery Condition." In Innovative Smart Grid Technologies Conference Europe (ISGT Europe), 2010 IEEE PES, 1-6.

Ota, Y, H Taniguchi, H Suzuki, T Nakajima, J Baba, and A Yokoyama. 2012. "Implementation of GridFriendly Charging Scheme to Electric Vehicle offBoard Charger for V2G," 1-6.

Oudalov, A., D. Chartouni, C. Ohler, and G. Linhofer. 2006. "Value Analysis of Battery Energy Storage Applications in Power Systems." In 2006 IEEE PES Power Systems Conference and Exposition, 2206-11. 
Oudalov, Alexandre, Rachid Cherkaoui, and Antoine Beguin. 2007. "Sizing and Optimal Operation of Battery Energy Storage System for Peak Shaving Application." In IEEE Lausanne Powertech Proceedings, 1-5.

Pandey, Shashi Kant, Soumya R. Mohanty, and Nand Kishor. 2013. "A Literature Survey on Loadfrequency Control for Conventional and Distribution Generation Power Systems." Renewable and Sustainable Energy Reviews 25 (September). Elsevier: 318-34. doi:10.1016/j.rser.2013.04.029.

Papi", Igor. 2006. "Simulation Model for Discharging a Lead-Acid Battery Energy Storage System for Load Leveling." IEEE Transaction on Energy Conversion 21 (2): 608-15.

Rahimi, A, M Zarghami, M Vaziri, and S Vadhva. 2013. "A Simple and Effective Approach for Peak Load Shaving Using Battery Storage Systems." In North American Power Symposium (NAPS), 2013, 1-5.

Ravi Shankar, Kalyan Chatterjee, and Ravi Bhushan. 2016. "Impact of Energy Storage System on Load Frequency Control for Diverse Sources of Interconnected Power System in Deregulated Power Environment." International Journal of Electrical Power \& Energy Systems 79: 11-26.

Rei, R J, F J Soares, P M Rocha Almeida, and J.a. Peças Lopes. 2010. "Grid Interactive Charging Control for Plug-in Electric Vehicles." In 2010 13th International IEEE Annual Conference on Intelligent Transportation Systems Madeira Island, Portugal, September 19-22, 2010, 386-91.

Ribeiro, Paulo F, Brian K Johnson, Mariesa L Crow, Aysen Arsoy, and Yilu Liu. 2001. "Energy Storage Systems for Advanced Power Applications." Proceedings of the IEEE 89 (12): 1744-56.

Ruegg, Rosalie, and Patrick Thomas. 2008. "Linkages of DOE's Energy Storage R\&D to Batteries and Ultracapacitors for Hybrid,Plug- In Hybrid, and Electric Vehicles." U.S. Department of Energy.

Samarakoon, Kamalanath, Janaka Ekanayake, and Nick Jenkins. 2012. "Investigation of Domestic Load Control to Provide Primary Frequency Response Using Smart Meters." IEEE Transactions on Smart Grid 3 (1): 282-92. doi:10.1109/TSG.2011.2173219.

Sasaki, T., T. Kadoya, and K. Enomoto. 2004. "Study on Load Frequency Control Using Redox Flow Batteries." IEEE Transactions on Power Systems 19 (1): 660-67. doi:10.1109/TPWRS.2003.818724.

Saxena, Sahaj, and Yogesh V Hote. 2013. "Load Frequency Control in Power Systems via Internal Model Control Scheme." IEEE Transaction on Power Systems 28 (3): 2749-57.

Sen, Umesh, and Narendra Kumar. 2014. "Load Frequency Control with Battery Energy Storage System." In Power, Control and Embedded Systems (ICPCES), 2014 International Conference on.
Serban, Ioan, and Corneliu Marinescu. 2014. "Control Strategy of Three-Phase Battery Energy Storage Systems for Frequency Support in Microgrids and with Uninterrupted Supply of Local Loads." IEEE Transaction on Power Electronics 29 (9): 5010-20.

Shayeghi, H., H.A. Shayanfar, and A. Jalili. 2009. "Load Frequency Control Strategies: A State-of-the-Art Survey for the Researcher." Energy Conversion and Management 50 (2): 344-53.

Shibasaki, So, Masato Toge, and Shinichi Iwamoto. 2012. "Load Frequency Control Using a NaS Battery System Controlled by a Kalman Filter." In 2012 10th International Power \& Energy Conference (IPEC), 236-41.

Shimizu, Koichiro, Taisuke Masuta, Yuyaka Ota, and Akihiko Yokoyama. 2010. "Load Frequency Control in Power System Using Vehicle-to-Grid System Considering the Customer Convenience of Electric Vehicles." In 2010 International Conference on Power System Technology, 1-8.

Short, Joe A., David G. Infield, and Leon L. Freris. 2007. "Stabilization of Grid Frequency through Dynamic Demand Control." IEEE Transactions on Power Systems $22 \quad$ (3): 1284-93. doi:10.1109/TPWRS.2007.901489.

Stock, Andrew, Petra Stock, and Veena Sahajwalla. 2015. "Powerful Potential: Battery Storage for Renewable Energy and Electric Cars." climatecouncil.org.au.

Subburaj, Anitha Sarah, and Stephen B Bayne. 2014. "Analysis of Dual Polarization Battery Model for Grid Applications." In Telecommunications Energy Conference (INTELEC), 2014 IEEE 36th International, 0-6.

Tan, Siew Chong, Chi Kwan Lee, and S. Y. Hui. 2013. "General Steady-State Analysis and Control Principle of Electric Springs with Active and Reactive Power Compensations." IEEE Transactions on Power Electronics 28 (8): 3958-69. doi:10.1109/TPEL.2012.2227823.

Tao, Zhanliang, and Jun Chen. 2015. "Distributed Demand Side Management with Energy Storage in Smart Grid." IEEE Transactions on Parallel and Distributed Systems 26 (12): 3346-57.

"Tesla Powerwall/ Home Battery." 2016. Accessed February https://www.teslamotors.com/powerwall.

Tullo, Alexander H. 2015. "Global Top 50 Chemical Companies." Chemical \& Engineering News. July. http://cen.acs.org/articles/93/i30/Global-Top50.html.

Umrao, Rahul, Sanjeev Kumar, Man Mohan, and D K Chaturvedi. 2012. "Load Frequency Control Methodologies for Power System." In 2012 2nd Nternational Conference on Power, Control and Embedded Systems. 
Vachirasricirikul, Sitthidet, and Issarachai Ngamroo. 2014. "Robust LFC in a Smart Grid with Wind Power Penetration by Coordinated V2G Control and." IEEE Transactions on Smart Grid 5 (1): 371-80.

Wada, Kohei, and Akihiko Yokoyama. 2012. "Load Frequency Control Using Distributed Batteries on the Demand Side with Communication Characteristics." In 2012 3rd IEEE PES Innovative Smart Grid Technologies Europe (ISGT Europe), 18. Ieee. doi:10.1109/ISGTEurope.2012.6465886.

$\mathrm{Xu}, \mathrm{Z}$., J. Ostergaard, M. Togeby, and C. MarcusMoller. 2007. "Design and Modelling of Thermostatically Controlled Loads as Frequency Controlled Reserve." In 2007 IEEE Power Engineering Society General Meeting, 1-6. Ieee. doi:10.1109/PES.2007.386014.

$\mathrm{Xu}$, Zhao, Jacob Østergaard, and Mikael Togeby. 2011. "Demand as Frequency Controlled Reserve." IEEE Transaction on Power Systems 26 (3): 1062-71.

Zhang, Liyan, Guo Chen, Zhuoyang Wang, Zhaoyang Dong, and David Hill. 2014. "Robust Hळ Load Frequency Control of Future Power Grid with Energy Storage Considering Parametric Uncertainty and Time Delay." In PES General Meeting Conference \& Exposition, 2014 IEEE.
Zhang, Shengqi, Yateendra Mishra, Ghavameddin Nourbakhsh, and Gerard Ledwich. 2013. "The Application of BESS in Load Shedding Scheme." In Australasian Universities Power Engineering Conference, 1-6.

Zhao, Changhong, Ufuk Topcu, and Steven H Low. 2012. "Frequency-Based Load Control in Power Systems." In 2012 American Control Conference, 4423-30.

Zhao, Changhong, Ufuk Topcu, and Steven H Low. 2013. "Optimal Load Control via Frequency Measurement and Neighborhood Area Communication." IEEE Transaction on Power Systems, 1-12. 